11 Michalak M, Groenendyk J, Szabo E, Gold LI, Opas M. Calreticulin, a multi-process calcium-buffering chaperone of the endoplasmic reticulum. Biochem J 2009; 417: 651-666.

12 Molinari M, Eriksson KK, Calanca V, Galli C, Cresswell P, Michalak M et al. Contrasting functions of calreticulin and calnexin in glycoprotein folding and ER quality control. Mol Cell 2004; 13: 125-135.

13 Marty C, Pecquet C, Nivarthi H, Elkhoury M, Chachoua I, Tulliez $M$ et al. Calreticulin mutants in mice induce an MPL-dependent thrombocytosis with frequent progression to myelofibrosis. Blood 2015. published ahead of print 25 November 2015; doi: 10.1182/blood-2015-11-679571.

14 Chachoua IPC, El-Khoury M, Nivarthi H, Albu RI, Marty C, Gryshkova V et al. Thrombopoietin receptor activation by myeloproliferative neoplasm associated calreticulin mutants. Blood 2015. published ahead of print 14 December 2015; doi: 10.1182/blood-2015-11-681932.

15 Passamonti F, Caramazza D, Maffioli M. JAK inhibitor in CALR-mutant myelofibrosis. N Engl J Med 2014; 370: 1168-1169.

\begin{abstract}
cc) $($ T) $\odot$ This work is licensed under a Creative Commons AttributionNonCommercial-NoDerivs 4.0 International License. The images or other third party material in this article are included in the article's Creative Commons license, unless indicated otherwise in the credit line; if the material is not included under the Creative Commons license, users will need to obtain permission from the license holder to reproduce the material. To view a copy of this license, visit http:// creativecommons.org/licenses/by-nc-nd/4.0/
\end{abstract}

\title{
OPEN
}

\section{Role of CD20 expression and other pre-treatment risk factors in the development of infusion-related reactions in patients with CLL treated with obinutuzumab}

Leukemia (2016) 30, 1763-1766; doi:10.1038/leu.2016.41

Infusion-related reactions (IRRs) elicited by therapeutic antibodies are commonly observed in the treatment of hematologic malignancies. In contrast to the currently approved type 1 anti-CD20 monoclonal antibodies (mAb) rituximab and ofatumumab, obinutuzumab is different by virtue of being a type 2 antiCD20 mAb with increased capacity to induce direct cell death. ${ }^{1-5}$ The fragment crystallisable (Fc) portion is glycoengineered (afucosylated), which increases its binding affinity to FcyRIIIA and FcyRIIIB, ${ }^{1,2}$ enhancing antibody dependent cellular cytotoxicity and phagocytosis with less complement activation.

In the CLL11 trial (NCT01010061, 'stage 2'), patients with previously untreated chronic lymphocytic leukemia (CLL) and co-morbidities were randomized to receive either rituximab or obinutuzumab administered in combination with chlorambucil. ${ }^{6,7}$ In the head-to-head comparison of the two antibody-containing regimens, obinutuzumab treatment resulted in a statistically significant improvement in efficacy endpoints. However, this improvement was accompanied by an increase in IRRs, with higher overall incidence and severity when compared with the rituximab-treated arm but largely limited to the first infusion. These IRRs had clinical importance resulting in a 7\% discontinuation rate in the obinutuzumab-treated cohort, compared with $<1 \%$ for rituximab. Predisposing biologic and clinical risk factors for IRRs are not well defined. In an attempt to better understand the profile of patients with CLL at particular risk of IRRs, we used this large clinical data set to perform a multivariate analysis.

Patients treated with a first infusion of obinutuzumab $(n=331)$ or rituximab $(n=326)$ were included. Plausible baseline risk factors were identified a priori and included parameters of disease burden, patient-specific factors such as age, concurrent medications and co-morbidities, FcyR genotype and baseline laboratory values. The primary outcome, development of IRR with the first infusion, was defined as the occurrence of related signs and symptoms during or within $24 \mathrm{~h}$ of administration.
Multivariable logistic regression models were fit to assess associations between patient characteristics and early IRR. The robustness of the model generated was then internally verified using bootstrapping techniques. A landmark analysis was performed to evaluate the impact of early IRR on progressionfree survival (PFS) in both groups and Kaplan-Meier curves were used to illustrate the estimated conditional progression-free probabilities. All statistical analyses were conducted in SAS (SAS Institute, Cary, NC, USA).

The incidence of any grade IRR with the first infusion (all grades) was $65 \%(214 / 331)$ in the obinutuzumab-treated cohort and $27 \%$ $(88 / 326)$ in the rituximab-treated cohort. Severe IRR (grade $\geqslant 3$ ) events were seen in $20 \%(65 / 331)$ of patients treated with obinutuzumab and $3 \%(10 / 326)$ in the rituximab arm; there were no fatal IRRs. The features at baseline associated with increased risk of developing an IRR from obinutuzumab or rituximab were: higher density of CD20 expression on CD19+CD5+ CLL cells, increased CD16 (FcyRIIIA) expression on circulating CD56+ natural killer (NK) cells, palpable splenomegaly, higher absolute lymphocyte count (ALC) in peripheral blood (PB), neutropenia, a higher affinity FcyR genotype (VV or VF versus FF) and the presence of an underlying respiratory co-morbidity. Odds ratios (ORs) and 95\% confidence intervals $(\mathrm{Cls})$, based on complete case analysis, are presented in Table 1.

Since IRRs were more frequent and severe in obinutuzumabtreated patients, we next focused on this group exclusively. If patients treated with rituximab were excluded from the data set, the variable that contributed most to the probability of developing an IRR was the density of CD20 expression on CLL cells in PB as expressed by the mean fluorescent intensity (MFI) generated by flow cytometric analysis pre-treatment (OR 3.6, 95\% Cl 1.6-7.9). In addition, MFI of CD16 (OR 2.2, 95\% Cl 0.8-5.6) on gated NK cells and the presence of a palpable spleen (OR 1.1, 95\% Cl 0.98-1.2) retained significance. Other variables including baseline neutrophil count, ALC, the presence of respiratory co-morbidities and FcyR genotype did not appear to influence outcome to the same degree when rituximab-treated patients were excluded.

To externally validate the importance of CD20 MFI as a risk factor, data from any available prior trials involving 
CLL patients treated with either rituximab or obinutuzumab where pre-treatment MFI CD20 was obtained $(n=935)$ were pooled (NCT01010061, ${ }^{6}$ NCT01414205, ${ }^{8}$ NCT01300247 (ref. 9) and NCT00281918 ${ }^{10}$ ). Only severe (grade $\geqslant 3$ ) IRRs were analyzed as grades 1 and 2 had not been collected uniformly in all the trials available. Results were standardized and plotted for those patients treated with either obinutuzumab or rituximab and those patients who experienced reactions were identified (Supplementary Figure 1). Patients with higher baseline expression CD20 on their CLL cells were at greater risk for severe grade early IRR, especially those patients treated with obinutuzumab $(P=0.02)$.

As MFIs for CD20 and CD16 are not standardized or routinely available outside the setting of clinical trials, we performed a separate analysis on the CLL11 data set excluding the immunophenotyping data, with a focus on the obinutuzumabtreated patients only. The resulting model was significant for the presence of trisomy 12 being associated with increased risk

Table 1. Risk factors identified by IRR prediction model using the CLL11 data set

\begin{tabular}{|c|c|c|c|c|}
\hline Risk factor & & $O R^{\mathrm{a}}$ & $95 \% \mathrm{Cl}^{\mathrm{a}}$ & $\begin{array}{l}\text { Significance retained with bootstrapping } \\
\qquad(10000 \text { simulations; \%) }\end{array}$ \\
\hline Randomization & $\begin{array}{l}\text { Obinutuzumab versus } \\
\text { rituximab }\end{array}$ & 8.4 & $5.2-13.4$ & 100 \\
\hline CD20 MFI ${ }^{\mathrm{b}}$ expressed on CLL cells at baseline & & 3.5 & $1.9-6.7$ & 98 \\
\hline $\begin{array}{l}\text { CD16 MFI } \\
\text { baseline }\end{array}$ & & 3.2 & $1.5-6.8$ & 97 \\
\hline Baseline neutrophil count ${ }^{\mathrm{b}}\left(\times 10^{9} / \mathrm{l}\right)$ & & 0.31 & $0.15-0.63$ & 87 \\
\hline FcyRIIIA genotype (position 158) & FV versus FF & 1.8 & $1.1-2.8$ & 80 (overall) \\
\hline FcyRIIIA genotype (position 158) & $\mathrm{V}$ versus $\mathrm{FF}$ & 2.3 & $1.1-5.2$ & \\
\hline $\begin{array}{l}\text { Splenomegaly (as measured by physical examination } \\
\text { in } \mathrm{cm} \text { below costal margin) }\end{array}$ & & 1.1 & $1.01-1.15$ & 84 \\
\hline Baseline $\mathrm{ALC}^{\mathrm{b}}\left(\mathrm{x} 10^{9} / \mathrm{l}\right)$ & & 1.8 & $1.03-3.0$ & 65 \\
\hline Presence of respiratory co-morbidity & Yes/no & 1.7 & $1.07-2.7$ & 73 \\
\hline
\end{tabular}

Abbreviations: ALC, absolute lymphocyte count; $\mathrm{Cl}$, confidence interval; $\mathrm{CLL}$, chronic lymphocytic lymphoma; IRR, infusion-related reaction; $\mathrm{MFI}$, mean fluorescence intensity; NK, natural killer; OR, odds ratio. ${ }^{\mathrm{a}}>1$ implies increased risk, $<1$ implies reduced risk for increasing values of risk factor. ${ }^{\mathrm{b}} \mathrm{Variables}$ were log transformed before modeling.

a

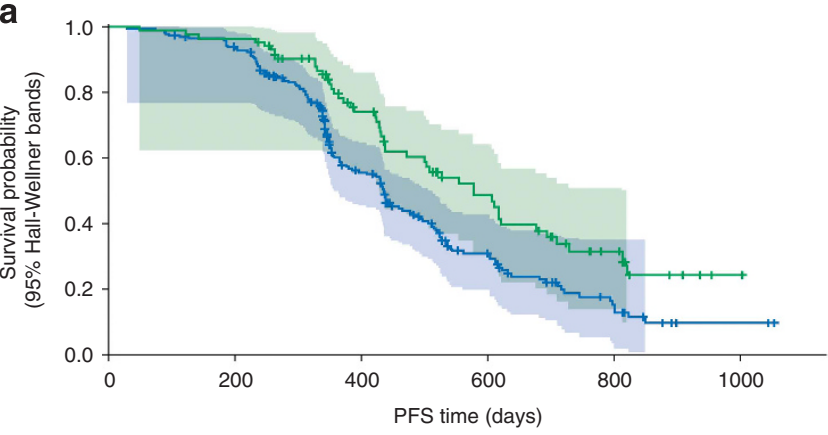

At risk: No 233

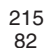

82

37
27
C

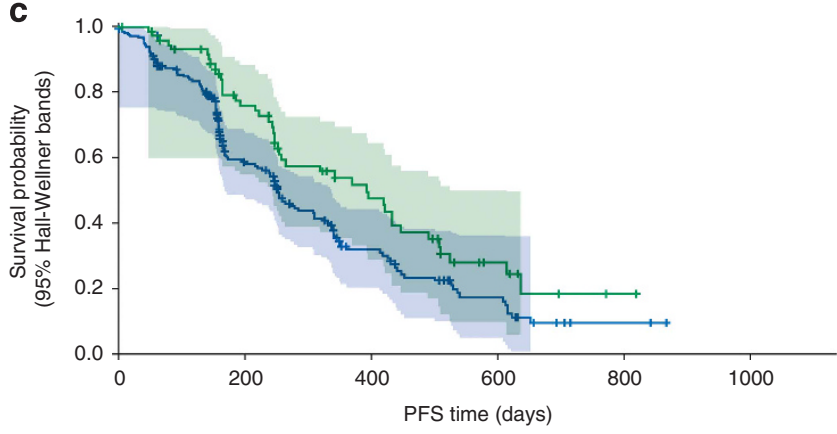

b

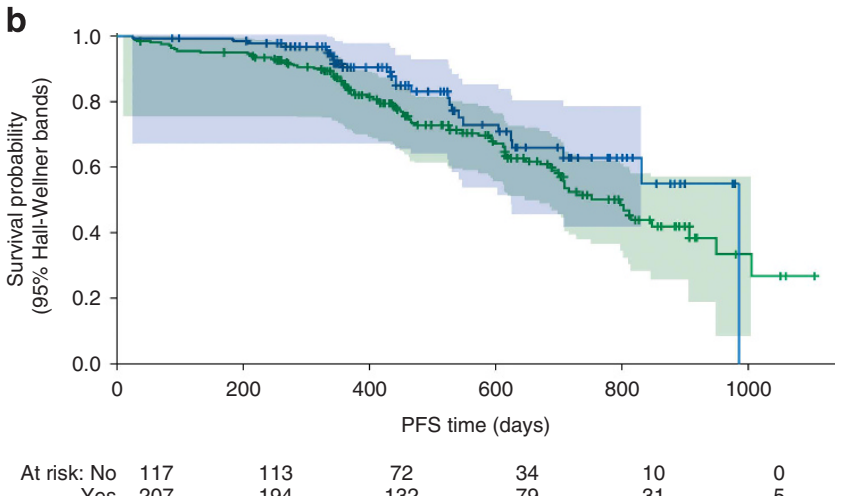

d

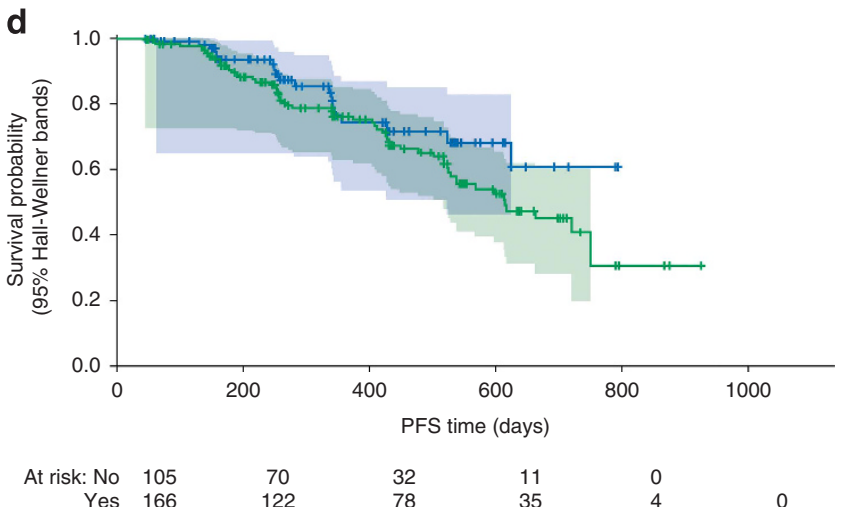

Figure 1. Prognostic significance of IRR in patients treated with anti-CD20 monoclonal antibodies. (a) Landmark analysis (starting day 3) of rituximab-treated patients. $\mathrm{HR}=0.6,95 \% \mathrm{Cl} 0.4-0.8, P=0.002$; (b) Landmark analysis (starting day 3 ) of obinutuzumab-treated patients. $\mathrm{HR}=1.5,95 \% \mathrm{Cl} 1.0-2.4, P=0.07$; (c) Landmark analysis of patients treated with 6 cycles of rituximab. $\mathrm{HR}=0.7,95 \% \mathrm{Cl} 0.5-0.9, P=0.01$; (d) Landmark analysis of patients treated with 6 cycles of obinutuzumab. $\mathrm{HR}=1.5,95 \% \mathrm{Cl} 0.9-2.6, P=0.1$. 
(OR 2.1, 95\% CI 1.02-4.2) along with splenomegaly (OR 1.1, 95\% $\mathrm{Cl} 1.0-1.2$ ) and baseline thrombocytopenia (OR 2.2, 95\% Cl 1.24.2). Of those patients with trisomy $12,41 / 55$ experienced an IRR $(P<0.05)$ compared with those without this cytogenetic abnormality.

To assess if the development of early IRR influenced outcome for patients treated with anti-CD20 mAbs, a landmark analysis was performed starting at study day 3 to eliminate the impact of patients who discontinued immediately with the first infusion. Prognostic factors at baseline that might otherwise influence PFS $^{11}$ were relatively balanced across all groups (Supplementary Table 1). For patients treated with rituximab (Figure 1a), there appeared to be a statistically significant benefit conferred, with improved PFS in those patients who experienced an early IRR (hazard ratio (HR) $0.6,95 \% \mathrm{Cl} 0.4-0.8, P=0.002$ ). For those patients treated with obinutuzumab, all patients had improved PFS compared with those treated with rituximab, but there appeared to be no benefit from having an early IRR in this cohort (HR 1.5, 95\% Cl 1.0-2.4, $P=0.07$ ) with no statistically significant difference between the two groups (Figures 1b). Including only patients that received all six cycles of therapy yielded similar results-a statistically significant benefit seen in those who experienced early IRR treated with rituximab (HR $0.7,95 \%$ $\mathrm{Cl} 0.5-0.9, P=0.01$ ) but no significant difference seen in those treated with obinutuzumab (HR 1.5, 95\% Cl 0.9-2.6, $P=0.1$; Figures $1 \mathrm{c}$ and $\mathrm{d}$ ).

This analysis, based on a large number of treatment-naïve CLL patients, has identified novel disease- and patient-specific biologic variables that appear to play a role in the development of IRR with anti-CD20 mAbs. Increased expression of CD20 on the surface of CLL cells at baseline was found to be a consistently important determinant of risk, a finding that we were able to validate with both internal and external data sets. When this variable was excluded for obinutuzumab-treated patients, the presence of Trisomy 12, known to be associated with higher levels of CD20 expression in $\mathrm{CLL}^{12,13}$ became statistically significant. In the absence of standardized methods of assessing and reporting CD20 MFI at the present time, this cytogenetic abnormality may prove a useful surrogate to identify patients at particular risk of IRR.

The risk factors of greatest importance would suggest that IRR result from enhanced binding of anti-CD20 biologics to both malignant B-cells and the FcyR of NK cells. The natural conclusion was that this would result in greater cytotoxicity and improvements in progression-free outcomes for patients. Increased levels of CD20 expression have been correlated with improved response to therapy, ${ }^{14}$ and for those patients treated with rituximab, the development of IRR was associated with improved response. However for patients treated with obinutuzumab, although PFS curves demonstrated consistent superiority over rituximab, the development of IRR did not appear to confer significant advantage in PFS in our analysis. The occurrence of IRR in rituximab-treated patients may well herald improved efficacy, whereas this improved efficacy was observed in all patients treated with the more potent type 2 antibody obinutuzumab, in whom the majority (66\%) experienced IRR. It is important to acknowledge that patients were not randomized to having an IRR and this hypothesis generating analysis will need to be further evaluated.

Our results support the idea that higher rates of IRR seen with the administration of obinutuzumab result from cellular activation and enhanced cross-linking between CD20 expressing leukemic cells and FcyRIIIA bearing effector cells-an on-target effect. As external validation was limited and models were derived using a single data set, these results will need to be corroborated with the generation of further patient data from ongoing clinical studies. The improvement in antibody technology realized by obinutuzumab can confer benefit to more patients if we can better understand how to predict, manage and prevent IRR. This is currently the subject of evaluation in ongoing clinical trials (NCT01905943 and NCT02336048) and these results may shed more light on how best to prevent this immunologic phenomenon.

\section{CONFLICT OF INTEREST}

CLF has received research funding, honoraria and other remuneration (travel, accommodation and expenses) from F. Hoffmann-La Roche Ltd. MD, RH, GF-R, KH, CSdC and VI are employees of F. Hoffmann-La Roche Ltd; MD and GF-R have stock ownership in F. Hoffmann-La Roche Ltd, which is publicly traded. K-AK has received consultancy fees, research funding, honoraria and paid expert testimony from F. Hoffmann-La Roche Ltd. SB has received consultancy fees and other remuneration (travel, accommodation and expenses) from AbbVie and F. Hoffmann-La Roche Ltd, research funding from AbbVie, Celgene Corporation and F. Hoffmann-La-Roche Ltd and honoraria from AbbVie, Becton Dickinson and F. Hoffmann-La Roche Ltd. SS has received consultancy fees and research funding from F. Hoffmann-La Roche Ltd/ Genentech Inc. JGG has received honoraria from Celgene Corporation, Gilead Sciences, F. Hoffmann-La Roche Ltd, Janssen-Cilag Ltd and Pharmacyclics LLC and other remuneration (travel, accommodation and expenses) from Gilead Sciences. $\mathrm{MHa}$ has received consultancy fees and research funding from, and served on a Speaker's Bureau for, F. Hoffmann-La Roche Ltd. VG has received consultancy fees and research funding from F. Hoffmann-La Roche Ltd, and honoraria from BristolMyers Squibb, F. Hoffmann-La Roche Ltd, GlaxoSmithKline and Mundipharma. MHe declares no conflicts of interest.

\section{ACKNOWLEDGEMENTS}

This research was sponsored by F. Hoffmann-La Roche Ltd. Statistical and programming support was provided by Nelson Kinnersley, Rosie Brown, Ute Dickschat and Renate Scheiner-Sparna. MH is supported by the Deutsche Forschungsgemeinschaft, KFO 826, TP6. Gardiner-Caldwell Communications, Macclesfield, UK, supported by funding from F. Hoffmann-La Roche Ltd, provided figure formatting and journal styling only to the authors during preparation of this manuscript.

\section{PREVIOUS PRESENTATIONS}

Data presented in part in abstract and poster format at the 56th American Society of Haematology Annual Meeting, San Francisco, December 2014 and the 55th Annual scientific meeting of the British Society of Haematology, April 2015. Abstracts were published per the below: Freeman $C L$, Dixon $M$, Houghton $R$, Humphrey K, Fingerle-Rowson G, Kreuzer K-A et al. Risk factors associated with the development of infusion-related reactions (IRRs) in patients with chronic lymphocytic leukaemia (CLL) treated with anti-CD20 monoclonal antibodies (mAbs) in combination with chlorambucil: analysis of the prospective, randomised phase III CLL11 study dataset. British Journal of Haematology 2015; 169: Abstract 152. Freeman C.L, Dixon M, Houghton R, Humphrey K, Fingerle-Rowson G, Kreuzer K-A et al. Risk Factors Associated with the Development of Infusion-Related Reactions in Patients with Chronic Lymphocytic Leukaemia Treated with Anti-CD20 Monoclonal Antibodies: Analysis of the CLL11 Study Dataset. Blood 2014; 124: Abstract 3339.

\section{AUTHOR CONTRIBUTIONS}

CLF, MD and GF-R designed the research; CLF and MD performed the research; $\mathrm{KH}, \mathrm{CSdC}, \mathrm{VI}, \mathrm{SS}, \mathrm{MHa}$ and VG collected the data; CLF, MD, RH, K-AK, GF-R, MHe, $\mathrm{SB}, \mathrm{MHa}, \mathrm{CSdC}, \mathrm{VI}$ and VG analyzed and interpreted the data; MD performed the statistical analysis and CLF wrote the manuscript. All authors reviewed the manuscript and approved the final version for submission.

CL Freeman ${ }^{1}, M$ Dixon ${ }^{2}$, R Houghton $^{3}$, K-A Kreuzer ${ }^{4}$, G Fingerle-Rowson ${ }^{5}$, M Herling ${ }^{4}$, K Humphrey $^{6}$, S Böttcher $^{7}$ CS de Costa $^{8}$, V Iglesias ${ }^{8}, S^{\prime}$ Stilgenbauer ${ }^{9}$, J Gribben ${ }^{1}$, M Hallek ${ }^{4,10}$ and V Goede ${ }^{4,10}$

${ }^{1}$ Department of Haemato-Oncology, John Vane Cancer Centre, Barts Cancer Institute, Queen Mary University of London, Charterhouse Square, London, UK;

${ }^{2}$ Department of Biostatistics, Roche Products Limited, Welwyn Garden City, UK;

${ }^{3}$ Department of Statistical Programming and Analysis, Roche Products Limited, Welwyn Garden City, UK; 
${ }^{4}$ German CLL Study Group, Center of Integrated Oncology CologneBonn, Department of Internal Medicine, University Hospital Cologne, Cologne, Germany;

${ }^{5}$ Pharma Development Oncology, F. Hoffmann-La Roche Ltd., Basel, Switzerland;

${ }^{6}$ Pharma Development Clinical Science, Roche Products Limited, Welwyn Garden City, UK;

${ }^{7}$ University Hospital of Schleswig-Holstein, Campus Kiel, Kiel, Germany;

${ }^{8}$ Roche Pharmaceutical Research and Early Development (pRED), Pharmaceutical Sciences (PS), Roche Innovation Centre Basel, Basel, Switzerland;

${ }^{9}$ Department of Internal Medicine III, UIm University, Ulm, Germany and ${ }^{10}$ Department I of Internal Medicine Centre of Integrated Oncology Köln Bonn and Cluster of Excellence on Cellular Stress Responses in Aging (CECAD) University of Cologne, Cologne, Germany E-mail: c.freeman@qmul.ac.uk

\section{REFERENCES}

1 Alduaij W, Ivanov A, Honeychurch J, Cheadle EJ, Potluri S, Lim SH et al. Novel type II anti-CD20 monoclonal antibody (GA101) evokes homotypic adhesion and actin-dependent, lysosome-mediated cell death in B-cell malignancies. Blood 2011; 117: 4519-4529.

2 Mössner E, Brünker P, Moser S, Püntener U, Schmidt C, Herter S et al. Increasing the efficacy of $C D 20$ antibody therapy through the engineering of a new type II anti-CD20 antibody with enhanced direct and immune effector cell-mediated B-cell cytotoxicity. Blood 2010; 115: 4393-4402.

3 Patz M, Isaeva P, Forcob N, Müller B, Frenzel LP, Wendtner CM et al. Comparison of the in vitro effects of the anti-CD20 antibodies rituximab and GA101 on chronic lymphocytic leukaemia cells. Br J Haematol 2011; 152: 295-306.

4 Herter S, Herting F, Mundigl O, Waldhauer I, Weinzierl T, Fauti T et al. Preclinical activity of the type II CD20 antibody GA101 (obinutuzumab) compared with rituximab and ofatumumab in vitro and in xenograft models. Mol Cancer Ther 2013; 12: 2031-2042.

5 Bologna L, Gotti E, Manganini M, Rambaldi A, Intermesoli T, Introna M et al. Mechanism of action of type II, glycoengineered, anti-CD20 monoclonal antibody GA101 in B-chronic lymphocytic leukemia whole blood assays in comparison with rituximab and alemtuzumab. J Immunol 2011; 186: 3762-3769.
6 Goede V, Fischer K, Busch R, Engelke A, Eichhorst B, Wendtner CM et al. Obinutuzumab plus chlorambucil in patients with CLL and coexisting conditions. N Engl J Med 2014; 370: 1101-1110.

7 Goede V, Fischer K, Engelke A, Schlag R, Lepretre S, Montero LF et al. Obinutuzumab as frontline treatment of chronic lymphocytic leukemia: updated results of the CLL11 study. Leukemia 2015; 29: 1602-1604.

8 Flynn JM, Byrd JC, Kipps TJ, Boxer M, Kolibaba KS, Tyson N et al. Obinutuzumab (GA101) $1,000 \mathrm{mg}$ versus $2,000 \mathrm{mg}$ in patients with chronic lymphocytic leukemia (CLL): Results of the phase II GAGE (GAO4768g) trial. J Clin Oncol 2014; 32: Abstract 7083.

9 Brown JR, O'Brien S, Kingsley CD, Eradat H, Pagel JM, Lymp J et al. Safety and efficacy of obinutuzumab (GA101) with fludarabine/cyclophosphamide (G-FC) or bendamustine (G-B) in the initial therapy of patients with chronic lymphocytic leukemia (CLL): results from the phase 1b Galton trial (GAO4779g). Blood 2013; 122: 523.

10 Hallek M, Fischer K, Fingerle-Rowson G, Fink AM, Busch R, Mayer J et al. Addition of rituximab to fludarabine and cyclophosphamide in patients with chronic lymphocytic leukaemia: a randomised, open-label, phase 3 trial. Lancet 2010; 376: 1164-1174.

11 Pflug N, Bahlo J, Shanafelt TD, Eichhorst BF, Bergmann MA, Elter T et al. Development of a comprehensive prognostic index for patients with chronic lymphocytic leukemia. Blood 2014; 124: 49-62.

12 Tam CS, Otero-Palacios J, Abruzzo LV, Jorgensen JL, Ferrajoli A, Wierda WG et al. Chronic lymphocytic leukaemia CD20 expression is dependent on the genetic subtype: a study of quantitative flow cytometry and fluorescent in-situ hybridization in 510 patients. Br J Haematol 2008; 141: 36-40.

13 Witzig TE, Borell TJ, Herath JF, Tefferi A, Li CY, Jenkins RB. Detection of trisomy 12 by FISH in untreated B-chronic lymphocytic leukemia: correlation with stage and CD20 antigen expression intensity. Leuk Lymphoma 1994; 14: 447-451.

14 Fang C, Zhuang Y, Wang L, Fan L, Wu YJ, Zhang R et al. High levels of CD20 expression predict good prognosis in chronic lymphocytic leukemia. Cancer Sci 2013; 104: 996-1001.

\section{(i) () $\Theta$}

This work is licensed under a Creative Commons AttributionNonCommercial-NoDerivs 4.0 International License. The images or other third party material in this article are included in the article's Creative Commons license, unless indicated otherwise in the credit line; if the material is not included under the Creative Commons license, users will need to obtain permission from the license holder to reproduce the material. To view a copy of this license, visit http:// creativecommons.org/licenses/by-nc-nd/4.0/

\section{Familial associations of monoclonal gammopathy of unknown significance with autoimmune diseases}

\section{Leukemia (2016) 30, 1766-1769; doi:10.1038/leu.2016.43}

Monoclonal gammopathy of undetermined significance (MGUS) is an asymptomatic premalignant clonal condition, which is diagnosed often fortuitously, through high levels of immunoglobulin-derived serum M-protein produced by a plasma cell clone. ${ }^{1,2}$ The prevalence of MGUS increases with age, and in predominantly white populations, it increases from $3 \%$ at age 60 years to $9 \%$ at age $80-89$ years. ${ }^{3}$ MGUS is a precursor condition for multiple myeloma (MM) and light chain amyloidosis, which are progressive conditions with a fatal outcome. Both MGUS and MM show familial clustering among and between these diseases, suggesting that they share genetic susceptibility; MGUS is also related to some other malignancies of the B-cell lineage. ${ }^{4-7}$ Known nongenetic risk factors of MGUS include prior infections and exposure to herbicides. ${ }^{8-10}$ Studies on the association of MGUS with personal or familial autoimmune diseases (ADs) have shown several associations for personal history, whereas for family history of combined ADs, the risk of 1.1 was reported. ${ }^{9,11}$ For MM, both personal and family histories with certain ADs have been identified. ${ }^{9,11,12}$ However, the risk for MM has been overall small (1.1), and found only in ankylosing spondylitis and systemic sclerosis patients. ${ }^{12}$

For the present study, we identified 12140 MGUS patients from Swedish nationwide hospital records, and from the same sources, we identified 769991 patients with any of 43 ADs. Combining these into a nationwide family register, we provide familial risk for MGUS patients whose first-degree relatives were diagnosed with ADs. Conversely, we also calculated risks for AD patients whose family members were diagnosed with MGUS to yield a largely independent risk estimate. Because of large sex-preference among $A D s$, sex-specific familial risks were also shown. 